\title{
Jahn, Robert W.
}

\section{Stützlehrer als neuer pädagogischer Profi in der Beruflichen Bildung?!}

Seifried, Jürgen [Hrsg.]; Faßhauer, Uwe [Hrsg.]; Seeber, Susan [Hrsg.]: Jahrbuch der berufs- und wirtschaftspädagogischen Forschung 2014. Opladen [u.a.] : Verlag Barbara Budrich 2014, S. 147-159. (Schriftenreihe der Sektion Berufs- und Wirtschaftspädagogik der Deutschen Gesellschaft für Erziehungswissenschaft (DGfE); 217)

Quellenangabe/ Reference:

Jahn, Robert W.: Stützlehrer als neuer pädagogischer Profi in der Beruflichen Bildung?! - In: Seifried, Jürgen [Hrsg.]; Faßhauer, Uwe [Hrsg.]; Seeber, Susan [Hrsg.]: Jahrbuch der berufs- und wirtschaftspädagogischen Forschung 2014. Opladen [u.a.] : Verlag Barbara Budrich 2014, S. 147-159 URN: urn:nbn:de:0111-pedocs-97325 - DOI: 10.25656/01:9732

https://nbn-resolving.org/urn:nbn:de:0111-pedocs-97325

https://doi.org/10.25656/01:9732

in Kooperation mit / in cooperation with:

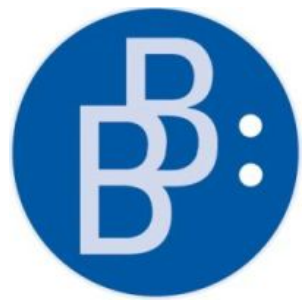

https://www.budrich.de

\section{Nutzungsbedingungen}

Dieses Dokument steht unter folgender Creative Commons-Lizenz:

http://creativecommons.org/licenses/by-nc-nd/3.0/de/deed - Sie dürfen das Werk bzw. den Inhalt unter folgenden Bedingungen vervielfältigen, verbreiten und öffentlich zugänglich machen: Sie müssen den Namen des Autors/Rechteinhabers in der von ihm festgelegten Weise nennen. Dieses Werk bzw. dieser Inhalt darf nicht für kommerzielle Zwecke verwendet werden und es darf nicht bearbeitet, abgewandelt oder in anderer Weise verändert werden.

Mit der Verwendung dieses Dokuments erkennen Sie die Nutzungsbedingungen an.

\section{Terms of use}

This document is published under following Creative Commons-License: http://creativecommons.org/licenses/by-nc-nd/3.0/de/deed.en - You may copy, distribute and transmit, adapt or exhibit the work in the public as long as you attribute the work in the manner specified by the author or licensor. You are not allowed to make commercial use of the work or its contents. You are not allowed to alter, transform, or change this work in any other way.

By using this particular document, you accept the above-stated conditions of use.

\section{Kontakt / Contact:}

\section{peDOCs}

DIPF | Leibniz-Institut für Bildungsforschung und Bildungsinformation Informationszentrum (IZ) Bildung

E-Mail: pedocs@dipf.de

Internet: www.pedocs.de

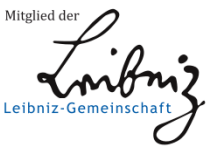




\section{Jahrbuch der berufs- und wirtschaftspädagogischen Forschung 2014}

Jürgen Seifried, Uwe Faßhauer Susan Seeber (Hrsg.)

DGE Deutsche Gesellschaft 
Schriftenreihe der Sektion

Berufs- und Wirtschaftspädagogik der Deutschen Gesellschaft für Erziehungswissenschaft (DGfE) 
Jürgen Seifried

Uwe Faßhauer

Susan Seeber (Hrsg.)

\section{Jahrbuch der berufs- und wirtschaftspädagogischen Forschung 2014}

Verlag Barbara Budrich

Opladen • Berlin • Toronto 2014 
Bibliografische Information der Deutschen Nationalbibliothek

Die Deutsche Nationalbibliothek verzeichnet diese Publikation in der Deutschen Nationalbibliografie; detaillierte bibliografische Daten sind im Internet über http://dnb.d-nb.de abrufbar.

(C) Dieses Werk ist im Verlag Barbara Budrich erschienen und steht unter folgender Creative Commons Lizenz: http://creativecommons.org/licenses/by-nc-nd/3.0/de/ Verbreitung, Speicherung und Vervielfältigung erlaubt, kommerzielle Nutzung und Veränderung nur mit Genehmigung des Verlags Barbara Budrich.

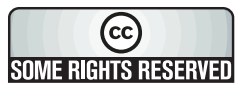

Dieses Buch steht im OpenAccess Bereich der Verlagsseite zum kostenlosen Download bereit (http://dx.doi.org/10.3224/84740164)

Eine kostenpflichtige Druckversion (Printing on Demand) kann über den Verlag bezogen werden. Die Seitenzahlen in der Druck- und Onlineversion sind identisch.

$$
\begin{array}{ll}
\text { ISBN } & 978-3-8474-0164-3 \text { (Paperback) } \\
\text { eISBN } & \mathbf{9 7 8 - 3 - 8 4 7 4 - 0 4 4 1 - 5 ~ ( e B o o k ) ~} \\
\text { DOI } & 10.3224 / 84740164
\end{array}
$$

Umschlaggestaltung: bettina lehfeldt graphic design, Kleinmachnow

Typografisches Lektorat: Judith Henning, Hamburg

Verlag Barbara Budrich, http://www.budrich-verlag.de 


\section{Inhaltsverzeichnis}

Vorwort 9

\section{Teil I: Perspektiven der historischen Berufsbildungsforschung}

Frank-Lothar Kroll

Möglichkeiten und Notwendigkeiten historiographischen Arbeitens in der Berufs- und Wirtschaftspädagogik........................................................ 1

Volker Bank, Annekathrin Lehmann

Theodor Franke. Sächsischer Pionier wirtschaftspädagogischen

Denkens in Deutschland

Marcel Schweder

Lehrerarbeit im Strafvollzug - Ein Diskurs aus historischer Sicht

\section{Teil II: Kompetenzmodellierung, -messung und -förderung}

Eveline Wittmann, Ulrike Weyland, Annette Nauerth, Ottmar Döring, Simone Rechenbach, Julia Simon, Iberé Worofka Kompetenzerfassung in der Pflege älterer Menschen - Theoretische und domänenspezifische Anforderungen der Aufgabenmodellierung 53

Simon Heinen, Martin Frenz, Christopher M. Schlick

Bildung für nachhaltige Entwicklung in der Gebäudeenergieberatung - Entwicklung eines Kompetenzmodells für die Förderung reflexiver Handlungsfähigkeit

Diana Stuckatz, Cornelia Wagner

Qualifizierungsangebote in der Pflegehilfe für Personen mit geringen Grundbildungskenntnissen - Empirische Studien zur Entwicklung von Lehr-Lern-Umgebungen und Arbeitsmaterialien. 81 


\section{Teil III: Gestaltung und Analyse von Lehr-Lern-Prozessen}

Eva Höpfer, Andrea Reichmuth, Doreen Holtsch, Franz Eberle

Wer sieht was? - Zum Umgang mit unterschiedlichen Sichtweisen auf

Unterricht am Beispiel des kaufmännischen Berufsschulunterrichts 95

Mandy Hommel

Sozial geteilte Reflexion - eine explorative Studie im

Mathematikunterricht. 109

Gerhard Minnameier, Rico Hermkes

„Kognitive Aktivierung“ und „konstruktive Unterstützung“ als Lehr-

Lern-Prozess-Größen - Eine Konzeption im rechnungswesen-

didaktischen Kontext

\section{Teil IV: Lehrerbildung und pädagogische Professionalität}

Nicole Kimmelmann, Johannes Lang

Lehramtsstudierende mit Migrationshintergrund und ihre

Schwierigkeiten an der Universität

Robert W. Jahn

Stützlehrer als neuer pädagogischer Profi in der Beruflichen Bildung?! .... 147

Sabrina Berg

Pädagogische Praxis und Reproduktion sozialer Ungleichheit - zur

Berücksichtigung sozialer Herkunft im Wirtschaftsunterricht..... 161

\section{Teil V: Perspektiven der Berufsbildungsforschung}

\section{Miriam Voigt}

Neo-institutionalistische und mikropolitische Prozesse in

Schulentwicklungsprojekten

Lara Forsblom, Lucio Negrini, Jean-Luc Gurtner \& Stephan Schumann

Lehrvertragsauflösungen und die Rolle der betrieblichen Auswahl von

Auszubildenden 
Marius R. Busemeyer

Organisierte Interessen, Parteipolitik und institutioneller Wandel im deutschen Berufsbildungssystem

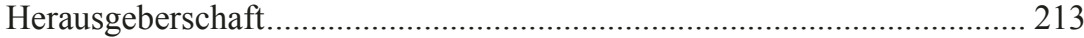

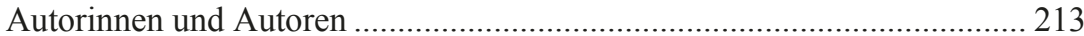




\section{Stützlehrer als neuer pädagogischer Profi in der Beruflichen Bildung?!}

Robert W. Jahn

\section{Bildungsträger und die Maßnahmen der Benachteiligtenförderung als Arbeitsmilieu ${ }^{1}$ von Stützlehrern}

Seitdem 1980 die Angebote der sozialpädagogisch orientierten Berufsausbildung (bzw. Regelangebote der Benachteiligtenförderung) konzeptionell entwickelt wurden, haben diese Maßnahmen sowie die beteiligten Organisationen und Akteure einen mehr oder minder erfolgreichen Prozess der Institutionalisierung durchlaufen. Seit 1988 sind sie gesetzlich fixiert und mittlerweile in den $\S \S 74 \mathrm{ff}$ SGB III verankert. In den vergangenen Jahren wurden jährlich durchschnittlich ca. 120.000 Jugendliche über ,ausbildungsbegleitende Hilfen“ $(\mathrm{abH})$ sowie die „Berufsausbildung in außerbetrieblichen Einrichtungen“ (BaE) gefördert (vgl. BiBB 2012, 238ff.).

Hinter diesen Maßnahmen steckt die Philosophie, dass die berufliche und gesellschaftliche Integration originär benachteiligter Jugendlicher nicht allein durch die Bereitstellung zusätzlicher Ausbildungsplätze gesichert werden kann, sondern es zudem einer spezifischen pädagogischen Förderung bedarf (vgl. Erbe/Vock 2003, S. 165). Denn gerade für diese Jugendliche ist die Aufnahme, das Durchhalten und v.a. der erfolgreiche Abschluss einer Berufsausbildung problematisch (vgl. Enggruber 2005). Die Maßnahmen sollen daher deren Chancen erhöhen, eine reguläre Berufsausbildung nach $\mathrm{BBiG} / \mathrm{HwO}$ erfolgreich zu absolvieren und in integrierte Beschäftigung überzugehen.

Die genannten Angebote sind durch verschiedene Spezifika auf pädagogisch-didaktischer, instutionell-organisatorischer und personeller Ebene gekennzeichnet. Auf pädagogisch-didaktischer Ebene sind sie durch die Didaktik der Benachteiligtenförderung als pädagogische „Spezialdisziplin“ der Berufs-, Sozial- und Sonderpädagogik (vgl. Biermann/Rützel 1999, S. 12) geprägt, die u.a. auf Prinzipien ganzheitlicher Lebensweltbezüge, eines Kompetenzansatzes, der Individualisierung und Partizipation beruht (vgl. BMBF 2005). Auf institutionell-organisatorischer Ebene ist hervorzuheben,

1 Dostal/Stooß/Troll (1998, S. 440) charakterisieren das Arbeitsmilieu als zentrales Merkmal von Berufen, das geprägt durch Wirtschaftszweig, Branche, spezifische Belastungen und besondere Arbeitsbedingungen als überorganisationales Arbeitsumfeld einzelne Berufe beeinflusst. 
dass Bildungsträger innerhalb dieser Maßnahmen einen gewichtigen Akteur und Lernort darstellen. Im Falle der BaE-Maßnahmen fungieren Träger als Ausbildender und übernehmen Funktionen der betrieblichen Ausbildung sodass hierdurch zusätzliche Ausbildungsplätze geschaffen werden. Daneben erfolgt beim Träger die sozialpädagogische Begleitung sowie Stütz- und Förderunterricht - als wesentliche Elemente der spezifischen Förderung der Auszubildenden. Darüber hinaus besuchen die Jugendlichen regulär die Berufsschule (vgl. BMBF 2005, S. 19ff.). Auf personeller Ebene agieren neben Ausbildern und Berufsschullehrern im Rahmen der Maßnahmen Sozialpädagogen sowie Lehrkräfte für den Stütz- und Förderunterricht (kurz: Stützlehrer) - als Angestellte der Träger. Innerhalb der Maßnahmen müssen somit pädagogische Fachkräfte integrativ zusammenwirken, die erstens sich hinsichtlich ihrer pädagogischen Sozialisationen erheblich voneinander unterscheiden, die zweitens ursprünglich nicht für den Einsatz in diesen Maßnahmen ausgebildet wurden (vgl. Christe et al. 2002) und die drittens besonderen Qualifikationsanforderungen unterliegen (vgl. Bojanowski 2008).

Ein weiteres Charakteristikum besteht hinsichtlich der Finanzierung, da die Träger den Auftrag für die Maßnahmen im Rahmen öffentlich-rechtlicher Vergabeverfahren von der Bundesagentur für Arbeit (BA) erhalten. Die Vergabepraxis hat durch das europäische Wettbewerbsrecht und im Zuge der Reorganisation der Arbeitsämter zur BA zu einer Dynamisierung und Verschärfung der Marktsituation für die Bildungsträger geführt. Der zunehmende Wettbewerb verursacht Konkurrenzdenken und Preisdruck (vgl. Grimm/ Vock 2007, S. 60ff.) und verringert Planungshorizonte. Dies führt zu z.T. prekären Beschäftigungsverhältnissen und Unsicherheiten (vgl. ebd., S. 56ff.), was die Attraktivität der Bildungsträger als Arbeitgeber mindert, Selbstselektionsmechanismen in Gang setzt und deprofessionalisierende Wirkungen entfaltet (vgl. BMBF 2005, S. 197).

Vor diesem Hintergrund fokussiert dieser Beitrag die Tätigkeit von Stützlehrern als pädagogische Spezialisierung, die seit ca. 30 Jahren besteht und von bis zu 10.000 Personen ausgeübt wird (vgl. Jahn 2012b, S. 73ff.), die bislang aber kaum systematisch betrachtet wurde (vgl. Sektion für Berufsund Wirtschaftspädagogik 2009, Jahn 2012b, S. 90f.). Das Ziel des Beitrags besteht darin, die Tätigkeit systematisch zu analysieren und zu beschreiben sowie ihren Status im Instituionalisierungsprozess zwischen Job und Profession zu bestimmen (vgl. Hartmann 1968). Dazu ist erstens die Funktion des Stützlehrers mithilfe berufsanalytischer Verfahren zu erfassen (vgl. Schanne 1990, Dostal/Stooß/Troll 1998) und zu prüfen, ob sie ebenso aus berufspädagogischer Perpektive als Beruf beschrieben werden kann (Kap. 2). Da Berufsanalysen immer auch die personalen Voraussetzungen einer Tätigkeit betrachten und diese im Falle pädagogischer Handlungsfelder vornehmlich aus einer professionwissenschaftlichen Perspektive diskutiert werden, erfolgt in Kap. 3 unter Einbezug soziologisch und psychologisch 
orientierter Modelle pädagogischer Professionalität eine Ableitung normativtheoretisch relevanter Dimensionen pädagogischer Professionalität. Dabei treten immer wieder Diskrepanzen zwischen normativen Forderungen und dem faktischen Zustand zutage, die auf die system-strukturellen Rahmenbedingungen zurückzuführen sind.

\section{Stützlehrer aus einer berufswissenschaftlichen Perspektive}

\subsection{Analysen beruflicher Aufgaben von Stützlehrern}

Im Weiteren werden zunächst drei berufswissenschaftlich orientierte Studien vorgestellt, die auf Ansätzen der Berufsforschung beruhen und der empirischsystematischen Erarbeitung objektiver und detaillierter Berufsbeschreibungen dienen (vgl. Schanne 1990, S. 112ff.). Mithilfe von Beobachtungs- und Befragungsverfahren sollen möglichst exakt Berufsinhalte ermittelt werden (vgl. ebd., S. 140). Bürgi (1976, S. 67f.) identifiziert Berufsziele, berufliche Aufgaben, Arbeitsabläufe und (organisations-)soziologische Rahmenbedingungen als Schwerpunkte der Berufsanalyse. Als theoretischer Referenzrahmen wird auf das Berufskonzept des IAB zurückgegriffen, nach dem ein Beruf durch ,alle für die Erledigung einer vorgegebenen Aufgabe notwendigen Merkmale in einer aufeinander abgestimmten Kombination" (Dostal/Stooß/Troll 1998, S. 440) bestimmt wird. Um den Beruf des Stützlehrers zu analysieren, ist es demnach erforderlich, diese Merkmale systematisch zu erfassen und zu beschreiben. Zwar stehen die beruflichen Aufgaben im Zentrum des Berufskonzeptes, jedoch ist es notwendig, erstens nach dem Funktionsbereich, Arbeitsmilieu und Arbeitsobjekt zu fragen. Dies wurde im einführenden Kapitel des Beitrags bereits vorweggenommen. Zweitens sind die beruflichen Ziele und Aufgaben zu identifizieren, die maßgeblich durch die erstgenannten Dimensionen determiniert werden. Daran anschließend stellen sich drittens Fragen nach dem Status sowie der Autonomie der Berufstätigen. Umrahmt wird das Berufskontrukt, von einem Spannungsfeld aus Anforderungen und Qualifikationen.

Aufgrund des unzureichenden und unsystematischen Forschungstands wurden drei Studien durchgeführt, die sich triangulierend ergänzen, indem sie aus multiplen Perspektiven mit unterschiedlichen Methoden die Berufsinhalte analysieren (s. Abb. 1). Innerhalb der ersten Erhebung wird sich trichterförmig aus verschiedenen Perspektiven ausgehend von normativen auf die faktischen Berufsinhalte zubewegt. Diese Befunde werden zu einem ersten Berufsprofil verdichtet, das in der zweiten Erhebung über eine standardisierte 
Befragung von Trägern und Beschäftigten validiert wird. Die dritte Erhebung dient der methodischen Triangulation zur Absicherung und Vertiefung der Befunde über ethnographisch orientierte Verfahren.

\section{Abb. 1: Mehrstufiges empirisches Vorgehen zur Aufgabenanalyse}

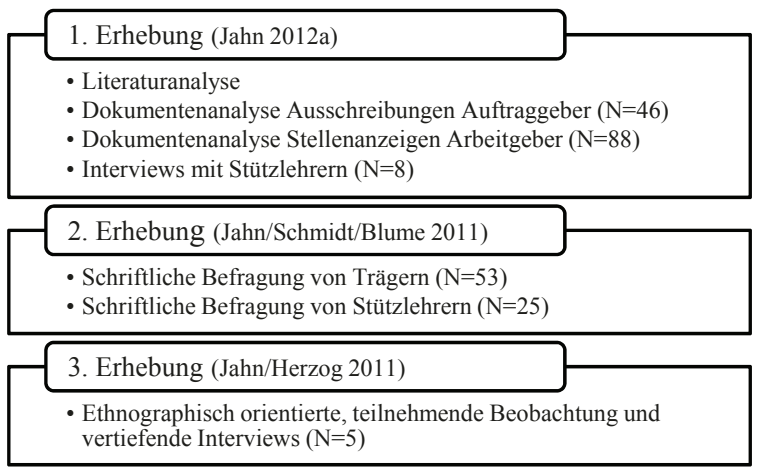

\section{Quelle: eigene Darstellung}

Die Analysen der ersten Erhebung verdeutlichen, dass aus verschiedenen Perspektiven (Science Community, Auftraggeber (BA), Arbeitgeber (Träger) und Beschäftigten) partiell differierende und z.T. unterschiedlich gewichtete Aufgaben beschrieben werden, die sich zu einem relativ kohärenten Kern verdichten lassen, der auch in den weiteren Analysen gültig bleibt (s. Abb. 2).

\section{Abb.2: Aufgaben von Stützlehrern im Überblick}

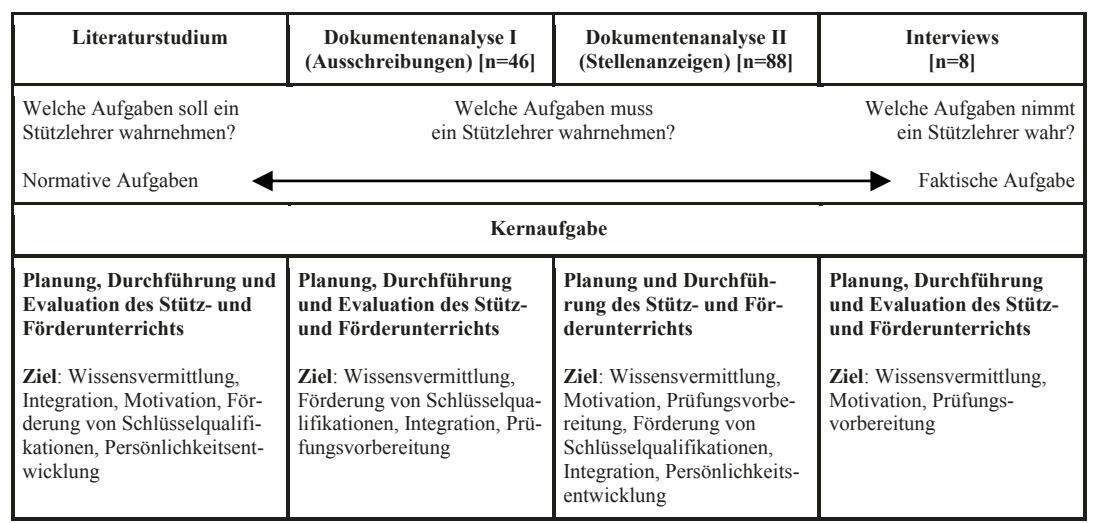

Quelle: Jahn 2012a, S. $199 f$. 
Abb.2: Aufgaben von Stützlehrern im Überblick (Fortsetzung)

\begin{tabular}{|c|c|c|c|}
\hline Literaturstudium & $\begin{array}{c}\text { Dokumentenanalyse I (Aus- } \\
\text { schreibungen) [ } \mathrm{n}=46]\end{array}$ & $\begin{array}{c}\text { Dokumentenanalyse II } \\
\text { (Stellenanzeigen) }[\mathrm{n}=\mathbf{8 8}]\end{array}$ & $\begin{array}{c}\text { Interviews } \\
\quad[\mathrm{n}=\mathbf{8}]\end{array}$ \\
\hline $\begin{array}{l}\text { Welche Aufgaben soll ein } \\
\text { Stützlehrer wahrnehmen? } \\
\text { Normative Aufgaben }\end{array}$ & \multicolumn{2}{|c|}{$\begin{array}{l}\text { Welche Aufgaben muss } \\
\text { ein Stützlehrer wahrnehmen? }\end{array}$} & $\begin{array}{r}\text { Welche Aufgaben nimmt } \\
\text { ein Stützlehrer wahr? } \\
\\
- \text { Faktische Aufgabe ung }\end{array}$ \\
\hline \multicolumn{4}{|c|}{ Querschnittsaufgaben } \\
\hline Lernberatung und -förderung & Lernberatung und -förderung & Lernberatung und -förderung & Lernberatung und -förderung \\
\hline \multirow[t]{2}{*}{$\begin{array}{l}\text { Diagnostische und curriculare } \\
\text { Aufgaben im Kontext der } \\
\text { Förderplanarbeit }\end{array}$} & $\begin{array}{l}\text { Diagnostische und curricula- } \\
\text { re Aufgaben im Kontext der } \\
\text { Förderplanarbeit }\end{array}$ & Curriculare Aufgaben & $\begin{array}{l}\text { Diagnostische und curricula- } \\
\text { re Aufgaben im Kontext der } \\
\text { Förderplanarbeit }\end{array}$ \\
\hline & Administrative Aufgaben & Administrative Aufgaben & Administrative Aufgaben \\
\hline Interne Kooperation im Team & $\begin{array}{l}\text { Interne Kooperation im } \\
\text { Team }\end{array}$ & $\begin{array}{l}\text { Interne Kooperation im } \\
\text { Team }\end{array}$ & $\begin{array}{l}\text { Interne Kooperation im } \\
\text { Team }\end{array}$ \\
\hline \multirow[t]{2}{*}{$\begin{array}{l}\text { Externe Kooperation mit Be- } \\
\text { rufs-schule und weiteren Insti- } \\
\text { tutionen der Benachteiligten- } \\
\text { förderung }\end{array}$} & $\begin{array}{l}\text { Externe Kooperation mit } \\
\text { Berufsschule und regionalen } \\
\text { Netzwerkpartnern }\end{array}$ & $\begin{array}{l}\text { Externe Kooperation mit } \\
\text { Berufsschule }\end{array}$ & $\begin{array}{l}\text { Externe Kooperation mit } \\
\text { Berufsschule }\end{array}$ \\
\hline & & $\begin{array}{l}\text { Selbstständige Wissens- } \\
\text { aneignung }\end{array}$ & $\begin{array}{l}\text { Selbstständige Wissens- } \\
\text { aneignung }\end{array}$ \\
\hline
\end{tabular}

Quelle: Jahn 2012a, S. $199 f$.

Die Kernaufgabe von Stützlehrern besteht in der Planung, Durchführung und Reflexion des Stütz- und Förderunterrichts. Dieser dient primär der Wissensvermittlung - und zwar sowohl im allgemeinbildenden als auch im fachtheoretischen Bereich, sodass eine enorme fachwissenschaftliche und fachdidaktische Breite erforderlich wird. Im Stütz- und Förderunterricht sollen Inhalte des Berufsschulunterrichts aufbereitet, Verständnisschwierigkeiten geklärt und alternative Repräsentationsmöglichkeiten angeboten sowie mangelhafte Grundlagenkenntnisse aufgearbeitet werden. Er zielt zudem auf die Motivation der Jugendlichen. Weiterhin ist eine wesentliche Zielstellung die Prüfungsvorbereitung, da das Bestehen der Prüfungen als primärer Erfolgsindikator seitens der Auftraggeber benannt wird. Nicht zuletzt hat er i.S.e. integrativen Konzeption der Maßnahmen erzieherische Funktionen - verbunden mit dem Ziel, die Persönlichkeitsentwicklung zu unterstützen.

Hinzu kommen Querschnittsaufgaben, die die Tätigkeit weiter spezifizieren. Hierzu gehören Lernberatung und -förderung - insbesondere auf einer affektiv-emotionalen Ebene, um Lernbereitschaften (wieder) zu wecken und Aversionen gegenüber Schule, Lernen und Lehrern aufzubrechen. Stützlehrer haben diagnostische Aufgaben, um Lernschwierigkeiten und -stände zu analysieren und darauf aufbauend, geeignete pädagogische Maßnahmen einzuleiten. Sie haben umfassende curriculare Aufgaben, da sie für jeden Jugendlichen individuell auf Basis der diagnostischen Informationen, in Abstim- 
mung mit Ausbildern, Sozialpädagogen und Berufsschullehrern, unter Partizipation der Jugendlichen und orientiert an Ausbildungsordnungen und Rahmenlehrplänen einen Förderplan (mit-)entwickeln müssen. Damit sind zum einen besondere Dokumentationspflichten angesprochen, über welche die BA die Maßnahmedurchführung kontrolliert. Zum anderen haben einrichtungsinterne sowie lernortübergreifende Kooperationsaufgaben eine hohe Bedeutung für das Handeln von Stützlehrern.

Letztlich sei informelles Lernen betont, da hieran verschiedene Probleme der Verberuflichung und Professionalisierung der Tätigkeit sichtbar werden. Denn vor dem Hintergrund der Aufgaben müssen Stützlehrer nicht nur - aus normativer Sicht - über sehr breite fachwissenschaftliche und fachdidaktische Inhalte verfügen, sondern sich diese z.T. selbst erarbeiten, da eine zielgerichtete, spezifische Vorbereitung auf eine solche Tätigkeit in entsprechenden Studiengängen faktisch kaum stattfindet, der Zugang zu formeller Weiterbildung begrenzt ist und Berufe und Berufsfelder, in denen sie eingesetzt werden, wechseln können, je nachdem in welcher Ausschreibung der Träger erfolgreich ist. Insgesamt werden anspruchsvolle pädagogische Aufgaben formuliert, mit denen hohen Anforderungen einhergehen (vgl. Jahn 2012b).

\subsection{Formale Qualifikationen und Beschäftigungsbedingungen}

Vor diesem Hintergrund fordert das BMBF (2005, S. 25) als Formalqualifikation ein 2. Staatsexamen in einem Lehramt. In den Maßnahmeausschreibungen der BA wird festgelegt, dass Stützlehrer „ein abgeschlossenes Fachhoch-/Hochschulstudium“ (vgl. z. B. Bundesagentur für Arbeit 2009, S. 16) haben sollen. Ein pädagogisches Studium wird jedoch nicht explizit erwartet. Ersatzweise wird eine Fachschulausbildung bzw. Meister- oder Fachwirtausbildung anerkannt (vgl. ebd.). Auch das BMBF (2005, S. 25) führt aus, dass Stützlehrer ,in begründeten Ausnahmefällen [...] im Endsemester der entsprechenden Studiengänge" sind, sodass die normativ hohe Formalqualifikation faktisch mit umfangreichen Ausnahmeregelungen versehen ist.

Zwar ermitteln Grimm/Vock (2007, S. 71f.) mit ca. 85\% einen relativ hohen Akademisierungsgrad von Stützlehrern, allerdings ist unklar, ob diese über ein pädagogisches Studium bzw. darüber hinaus das 2. Staatsexamen in einem Lehramt verfügen. In entsprechenden Stellenanzeigen werden bspw. nur in $80 \%$ der Annoncen ein Hochschul- oder Fachhochschulstudium, in nur $51 \%$ explizit ein „pädagogisches“ Studium und in $27 \%$ ausschließlich ein Studium als Formalqualifikation gefordert. Das heißt, es werden häufig nichtakademische Formalqualifikationen angegeben, welche die Aufnahme einer Stützlehrertätigkeit (,ausnahmsweise“) ermöglichen sollen (vgl. Jahn 2012b, S. 170f.). Diese Anhäufung unterschiedlichster Formalqualifikationen kann ein Indiz dafür sein, dass das Qualifikationsbündel „Stützlehrer“ zum einen 
noch relativ unbestimmt ist und dass die Stützlehrertätigkeit zum anderen in bestehenden Ausbildungsgängen nicht hinreichend abgebildet wird. Zwar signalisieren Träger ein Interesse, formal qualifiziertes Personal einzustellen, allerdings öffnen sie sich und die Tätigkeit für nicht-akademisch und -pädagogisch ausgebildete Personengruppen. Dies könnte mit Kostenkalkülen sowie der Verfügbarkeit geeigneter Bewerber (aufgrund der Attraktivität der Beschäftigungsbedingungen) in Zusammenhang stehen.

Grimm/Vock (2007, S. 83 ff.) zeigen in diesem Kontext, dass die Reorganisation der Vergabepraxis und der zunehmende Wettbewerb zur Verschlechterung der Beschäftigungsbedingungen führten. Im Hinblick auf Befristungen kann festgestellt werden, dass die Zahl unbefristeter Verträge im Zeitraum 2004-2005 weiter abnahm (35,9\%) (vgl. ebd.). Bei Neueinstellungen werden sogar nur 14\% der Stellen unbefristet angeboten (vgl. Jahn 2012b, S. 164). Es ist offensichtlich, dass die Beschäftigungszeiträume an die Maßnahmelaufzeiten gekoppelt werden und dass das Feld von kurzfristigen Planungshorizonten und unsicheren Beschäftigungsperspektiven geprägt ist. Auch bezüglich des Gehalts vermuten Grimm/Vock (2007, S. 86ff.), dass die Träger im Zuge des forcierten Wettbewerbs kaum in der Lage sind, sich an Vergütungen von Lehrkräften im öffentlichen Dienst zu orientieren. Dies macht das Feld für formal hochqualifizierte pädagogische Fachkräfte unattraktiv, erklärt eine hohe Fluktuation und wird - so ist zu befürchten - sich langfristig negativ auf Qualitätsstandards und die Professionalisierung dieser Tätigkeit auswirken.

\subsection{Stützlehrer zwischen Job und Beruf}

Die berufsanalytischen Studien liefern eine empirisch fundierte Basis, die eine Beschreibung dieser Tätigkeit als Beruf zulässt. Die Befunde zu normativen und faktischen Aufgaben sowie weiteren Merkmalen (z.B. Qualifikationen, Beschäftigungsbedingungen) verdeutlichen, dass die systemstrukturellen Zwänge den Institutionalisierungsprozess der Tätigkeit - die vor dem Hintergrund der identifizierten anspruchsvollen pädagogischen Aufgaben normativ erforderlich erscheint - faktisch hemmen.

Versucht man die Tätigkeit von Stützlehrern im Spektrum möglicher Organisationsformen von Arbeit zwischen Job und Beruf zu verorten, ist zunächst festzuhalten, dass es sich bei der Tätigkeit von Stützlehrern nicht um einen Job - als ,voraussetzungslose, schnell zu lernende Teilaufgabe definiert" (Dostal/Stooß/Troll 1998, S. 440) - handelt. Vielmehr kann sie mit dem Berufskonstrukt des IAB als Beruf analysiert und beschrieben werden. Ebenso kann die Arbeit von Stützlehrern als Beruf bezeichnet werden, wenn man weitere berufssoziologisch oder -pädagogisch orientierte Definitionen zugrunde legt, da sie ,gesellschaftlichen Aufgabencharakter [...] [hat, R.J.] 
zu deren Bewältigung besondere Fähigkeiten, Fertigkeiten und Kenntnisse erworben werden müssen" (Huisinga/Lisop 1999, S. 17). Unter Bezug auf Beck/Brater/Daheim (1980, S. 20) stellt die Tätigkeit ein spezialisiertes Muster von Arbeitskraft dar, das von weiteren Tätigkeiten (z.B. Ausbilder, Sozialpädagogen, Berufsschullehrer etc.) in der Beruflichen Integrationsförderung abgrenzbar ist, als Ware am Arbeitsmarkt gehandelt wird und gegen Bezahlung in einem kooperativ organsierten Arbeitszusammenhang zum Einsatz kommt. Damit sind zentrale Aspekte gegeben, die eine Entwicklung neuer beruflicher Tätigkeitsstrukturen und erforderlicher Arbeitskraftmuster ermöglichen (vgl. ebd.) und die Chancen für eine Institutionalisierung des Berufs in Form der Herausbildung von Berufsbildern, Zertifikaten, Qualifizierungen, Berufsverbänden o.ä. (vgl. Bals et al. 2001, S. 14f.) eröffnen.

\section{Stützlehrer aus professionstheoretischer Perspektive}

\subsection{Stützlehrer im Lichte professionssoziologischer Ansätze}

Im Laufe des weiteren Institutionalisierungsprozesses von Tätigkeiten kann dann entsprechend des Ansatzes von Hartmann (1968) ein stetiger Übergang vom Beruf zur Profession erfolgen oder aber es finden entgegengesetzte Prozesse statt. Hier knüpft eine professionstheoretische Analyse an. Es besteht weitgehend Einigkeit darüber, dass die Professionalisierung des Personals in diesem Feld vorangetrieben werden sollte (vgl. Sektion Berufs- und Wirtschaftspädagogik 2009) und dass pädagogisches Handeln zumindest als professionalisierungsbedürftig (vgl. Oevermann 1996) charakterisiert wird. Aus professionstheoretischer Perspektive ist zunächst zu fragen, ob die Stützlehrertätigkeit faktische Merkmale einer Profession aufweist bzw. ob sie normativ professionalisierungsbedürftig ist.

Aus einer kriterienorientierten Perspektive professionssoziologischer Ansätze (vgl. Hesse 1972) muss man festhalten, dass Stützlehrer keine Profession darstellen. Auch wenn ihnen durch die Gesellschaft die Zuständigkeit zur Wahrung gesellschaftlicher Werte übertragen wurde, ist bspw. ihre Autonomie problematisch zu bewerten, da ihre pädagogische Tätigkeit zwar sehr offen definiert ist, sie die system-strukturellen Rahmenbedingungen jedoch kaum beeinflussen können. Zudem bestehen - wie oben gezeigt - keine klare Regelung des Zugangs und der Qualifikation, kein kodifizierter Wissenskorpus, kein Berufsverband und kein soziales Prestige als Experte.

Gleichsam ist diese Tätigkeit aber professionalisierungsbedürftig. $\mathrm{Zu}$ diesem Schluss führen strukturtheoretische und interaktionstheoretische Ansätze (vgl. Oevermann 1996, Schütze 1996, Helsper 1996), da die Tätigkeit ebenso 
wie andere pädagogische Tätigkeiten - vielleicht sogar in besonderem Maße - durch einen Balanceakt zwischen Distanz und Nähe, durch quasi-therapeutische Funktionen und die fall- und problembezogene Bearbeitung von Lebensproblemen ihrer Klienten geprägt ist, deren psychosoziale Integrität und gesellschaftliche Teilhabe ohne professionelle Unterstützung gefährdet ist. Ein solcher Professionalisierungsprozess ist u.a. durch eine Systematisierung und Kodifizierung des beruflichen/professionellen Wissens geprägt (vgl. Hartmann 1968). Für den Beruf „Stützlehrer“" wurde jedoch festgestellt, dass eine solche Systematisierung bislang weder hinreichend erarbeitet wurde, noch kodifiziert vermittelt oder in formalen Qualifikationen abgebildet wird.

\subsection{Stützlehrer im Lichte wissenspsychologisch orientierter Ansätze - Ein Modell pädagogischer Professionalität}

Wenn die Tätigkeit von Stützlehrern pädagogische Professionalität verlangt, d.h. eine besondere Qualität des Handelns sowie entsprechende personale Voraussetzungen, ist - sowohl aus berufs- als auch aus professionswissenschaftlicher Perspektive - zu analysieren, wie diese beschrieben werden kann. Problematisch ist dabei, dass aus empirisch erfassten Aufgaben nicht linear auf notwendige Kompetenzen geschlossen werden kann. Erst durch eine Verbindung des Aufgabenprofils, der Anforderungen der Benachteiligtendidaktik, dem heuristischen Professionalisierungskonzept von Bojanowski (2008) sowie der (wissenspsychologisch orientierten) professionstheoretischen Ansätze - v.a. von Oser (1997), Baumert/Kunter (2006), Arnold/Gomez-Tutor (2007) und Bauer (2005) - kann ein normativ-theoretisches Strukturmodell pädagogischer Professionalität von Stützlehrern konstruiert werden. Es leitet sich aus einem pädagogischen Handlungsmodell der Stützlehrertätgkeit (vgl. Jahn 2012b, S. 249 ff.) ab und verbindet über einen heuristischen Zugriff sieben Dimensionen: pädagogisches Ethos, motivationale Orientierungen, Reflexion und Lernen, selbstregulative Fähigkeiten, pädagogische Überzeugungen, erarbeitete Identität und professionelles Wissen und Können. Alle Dimensionen lassen sich in weitere Subdimensionen ausdifferenzieren, die theoretisch und/oder empirisch (wenn auch nur selten domänenspezifisch) begründet werden können und für das Handeln relevant sind - d.h. partiell Charakteristika von Standards nach Oser (1997) aufweisen.

Betrachtet man allein die einzelnen Facetten der Dimension des Wissens (vgl. Jahn 2012, S. 271-304, Abb. 3), wird deutlich, wie anspruchsvoll die formulierten personalen Voraussetzungen sind und dass begründet angenommen werden muss, dass sich diese nicht en passent allein im Kontext beruflichen Handelns entwickeln. Zudem wird hieran exemplarisch deutlich, wie die Systemarchitektur bis auf diese Ebene wirkt. Zum einen erfordert die 
maßnahmenspezifische Aufgaben- und Zielstruktur eine Breite an fachwissenschaftlichem und fachdidaktischem Wissen, die allgemein- und berufsbildende Domänen zugleich anspricht und dadurch nahezu unspezifisch bleibt. Zum anderen ist curriculares sowie Beratungs- und Organisationswissen überaus bedeutsam, das aufgrund der institutionell-organisatorischen, pädagogisch-didaktischen und personellen Besonderheiten der Benachteiligtenförderung sowie der Jugendlichen eine sehr spezifische Konnotation erfährt.

\section{Abb. 3: Subdimensionen professionellen Wissens von Stützlehrern}

\begin{tabular}{|c|c|c|}
\hline $\begin{array}{l}\text { Fachliches Wissen } \\
\text { - Wissen über berufliche Handlungsfelder } \\
\text { - Berufsfeldbezogenes fachtheoretisches } \\
\text { Wissen } \\
\text { - Fachwissen Deutsch und Mathematik } \\
\text { Allgemein-pädagogisches Wissen } \\
\text { - Wissen über das System der Beruflichen } \\
\text { Integrationsförderung } \\
\text { - Entwicklungspsychologisches Wissen } \\
\text { - Pädagogisch-psychologisches Wissen } \\
\text { Methodenwissen, Wissen über die } \\
\text { Konstruktion von Lernsituationen } \\
\text { - Allgemeindidaktisches Planungs- und } \\
\text { Konzeptionswissen } \\
\text { - Wissen über Unterrichtsführung und } \\
\text { Orchestrierung von Lerngelegenheiten } \\
\text { Fachdidaktisches Wissen } \\
\text { - Wissen über multiple Repräsentationen } \\
\text { fachtheoretischer Inhalte } \\
\text { Wissen bez. der Konzeption, Analyse, } \\
\text { Auswahl, Differenzierung und } \\
\text { Sequenzierung von Aufgaben } \\
\text { - Wissen über den Umgang mit Fehlern } \\
\text { Wissen über typische Schülerfehler }\end{array}$ & $\begin{array}{l}\text { Beratungswissen } \\
\text { r Wissen über Beratungstheorien, } \\
\text {-modelle und entsprechende empiri- } \\
\text { sche Forschung } \\
\text { - Beratungsprozesswissen und Wissen } \\
\text { über beratungsspezifische Kommu- } \\
\text { nikation } \\
\text { Beratungsobjektwissen, v.a. im Hin- } \\
\text { blick auf die Lernberatung } \\
\text { Curriculares Wissen } \\
\text { - Wissen über berufliche Handlungs- } \\
\text { felder } \\
\text { - Wissen über Prinzipien der Curricu- } \\
\text { lumrezeption und -konstruktion } \\
\text { - Wissen über Prinzipien der Förder- } \\
\text { planarbeit } \\
\text { Diagnostisches Wissen } \\
\text { - Wissen über Identifikation von } \\
\text { Lernstrategien, -stilen, und -typen } \\
\text { - Wissen über die Diagnose von } \\
\text { „Lernkrankheiten“ und Entwick- } \\
\text { lungsstörungen } \\
\text { - Wissen über Möglichkeiten der } \\
\text { Lernerfolgskontrolle } \\
\text { Wissen über Evaluationsmethoden }\end{array}$ & $\begin{array}{l}\text { Organisationswissen } \\
\text { - Wissen über Aufgaben, Zuständig- } \\
\text { keiten und Überzeugungen der ver- } \\
\text { schiedenen pädagogischen Akteure } \\
\text { und der Leitung } \\
\text { - Wissen über Dokumentations- und } \\
\text { Evaluationsinstrumente } \\
\text { Wissen über Konfliktpotentiale und } \\
\text { Lösungsmöglichkeiten } \\
\text { - Wissen über die kooperative Ent- } \\
\text { wicklung einer eigenen Förderphi- } \\
\text { losophie und deren integrative Um- } \\
\text { setzung } \\
\text { Wissen über Lernortkooperationen } \\
\text { und die Nutzung regionaler Netz- } \\
\text { werke } \\
\text { Wissen über rechtliche Rahmenbe- } \\
\text { dingungen der Beruflichen Integra- } \\
\text { tionsförderung sowie Anspruchs- } \\
\text { gruppen und deren Erwartungen }\end{array}$ \\
\hline
\end{tabular}

Quelle: eigene Darstellung

\section{4. (De-)Professionalisierung zwischen idealistischen Anforderungen und faktischen Rahmenbedingungen}

Mit diesem Strukturmodell wurde ein Idealtypus konstruiert, der einerseits in dieser Form überhöhte Ansprüche sowohl an die Beschäftigten als auch an Lehrerbildung stellt und - würde man Einzelne daran messen - wahrscheinlich zu einer künstlich konstruierten Defizit-Sicht führt. Eine realistische Wendung eines solchen Ideals ist allerdings erst auf empirischer Basis zu leisten. Dazu bedarf es eines Orientierungsrahmens, d.h. es ist zunächst zu analysieren, welche Dimensionen pädagogischer Professionalität für das 
Handeln von Stützlehrern relevant sein sollten, bevor diese im Hinblick auf ihre Ausprägungen, Wirkungen und Entwicklung untersucht werden können. Andererseits ist die Beschreibung eines Ideals angesichts der drohenden Konsequenzen gescheiterter Integrationsbemühungen für Gesellschaft, Wirtschaft und v.a für die Jugend angemessen, da die pädagogische Arbeit mit Benachteiligten nicht durch die Definition kurzfristig finanzierbarer und disponibler Mindestqualifikationsanforderungen geprägt werden sollte. Vielmehr gilt angesichts der schwierigen Lage der Jugendlichen: „Das Beste ist gerade gut genug" (Nieke 2002, S. 16).

Auch wenn die Analysen auf einen Status quo bezogen waren, wird im Hinblick auf Verberuflichungs- und Professionalisierungsprozesse deutlich, dass der system-strukturelle Rahmen hier negative Wirkungen entfaltet, da er die Maßnahmen als pädagogisches Betätigungsfeld unattraktiv macht und eine Systematisierung und Verwissenschaftlichung der Wissensbasis sowie die Etablierung adäquater Ausbildungsstrukturen hemmt (vgl. Hartmann 1968). Gerade dies wäre aber im Hinblick auf die Professionalisierung von Stützlehrern relevant, da künftige Berufstätige dann weniger in diesen Beruf hineinschlittern würden, sondern sich frühzeitig und bewusst für eine Ausbildung und diesen Beruf entscheiden. Anhand dieser Erkenntnisse werden auch offene Fragen der Weiterentwicklung des Systems der Beruflichen Integrationsförderung, generelle Fragen der Ausdifferenzierung und Spezialisierung pädagogischer Berufe sowie der Professionalisierung pädagogischer Tätigkeiten jenseits staatlicher Schulen deutlich.

Angesichts des anhaltenden Problems der schwierigen beruflichen und gesellschaftlichen Integration Benachteiligter besteht auf praktischer Ebene die Notwendigkeit, mehr Anstrengungen zu unternehmen, um den vielleicht „schwierigsten“ und gefährdetsten Jugendlichen die „besten“ - zumindest professionell ausgebildete - Pädagogen an die Seite zu stellen und sie nicht allein ihrem Schicksal zu überlassen. Dazu wären die Förderstrukturen des System so zu verändern, dass jenseits der New-Public-Management-Logik nachhaltige und sichere Strukturen geschaffen werden, die Anreize setzen, damit Wissenschaft, BA, Träger sowie die Beschäftigten spezifische Investitionen in die Professionalisierung dieser Akteure tätigen. An die Berufstätigen wäre $\mathrm{zu}$ appellieren, berufskollektive Vereinigungen $\mathrm{zu}$ gründen, um professionelle Aufstiegsprojekte zu initiieren. 


\section{Literatur}

Arnold, R./Gómez Tutor, C. (2007): Grundlinien einer Ermöglichungsdidaktik. Augsburg: ZIEL.

Bals, T./Brater, M./Hemmer-Schanze, C./et al. (2001): In vier Stufen zum Beruf. In: Bullinger, H.-J. (Hrsg.): Gesundheit, Wellness, Wohlbefinden. Bielefeld: Bertelsmann, S. 11-76.

Bauer, K.-O. (2005): Pädagogische Basiskompetenzen. Weinheim u.a.: Juventa.

Baumert, J./Kunter, M. (2006): Stichwort: Professionelle Kompetenz von Lehrkräften. In: Zeitschrift für Erziehungswissenschaft, 9. Bd., H. 4, S. 469-520.

Beck, U./Brater, M./Daheim, H. (1980): Soziologie der Arbeit und der Berufe. Reinbek bei Hamburg: Rowohlt.

BiBB (2012): Datenreport zum Berufsbildungsbericht 2012. Bonn: BiBB.

Biermann, H./Rützel, J. (1999): Didaktik der beruflichen Bildung Benachteiligter. In: Biermann, H./Bonz, H./Rützel, J. (Hrsg.): Beiträge zur Didaktik der Berufsbildung Benachteiligter. Stuttgart: Holland + Josenhans, S. 11-37.

BMBF (2005): Berufliche Qualifizierung Jugendlicher mit besonderem Förderbedarf. Bonn, Berlin: BMBF.

Bojanowski, A. (2008): Professionalisierung des Fachpersonals in der beruflichen Benachteiligtenförderung. In: Faßhauer, U./Münk, D./Paul-Kohlhoff, A. (Hrsg.): Berufspädagogische Forschung in sozialer Verantwortung. Stuttgart: Steiner, S. 109-220.

Bundesagentur für Arbeit (2009): Verdingungsunterlagen zur öffentlichen Ausschreibung der Berufsausbildung in einer außerbetrieblichen Einrichtung - integratives Modell. Vergabenummer: 701-09-B§242-00041 (Unveröff. Manuskript).

Bürgi, A. (1976): Die Analyse von Berufen und Berufsanforderungen. Stuttgart u.a.: Kohlhammer.

Christe, G./Enggruber, R./Fülbier, P./et al. (2002): Benachteiligtenförderung und Fachhochschulen. Oldenburg: IAJ.

Dostal, W. (2002): Der Berufsbegriff in der Berufsforschung des IAB. In: Kleinhenz, G. (Hrsg.): IAB-Kompendium Arbeitsmarkt- und Berufsforschung. Nürnberg: IAB, S. 463-474.

Dostal, W./Stooß, F./Troll, L. (1998): Beruf - Auflösungstendenzen und erneute Konsolidierung. In: Mitteilungen aus der Arbeitsmarkt- und Berufsforschung, 31. Bd., H. 3, S. 438-460.

Enggruber, R. (2005): Zur Vielfalt benachteiligter junger Menschen - ein Systematisierungsversuch. In: Berufsbildung, 59. Bd., H. 93, S. 35-37.

Erbe, B./Vock, R. (2003): Der besondere Weg zum Beruf. In: Bonifer-Dörr, G./Vock, R. (Hrsg.): Berufliche Integration junger Menschen mit besonderem Förderbedarf. Darmstadt: Hiba, S. 151-179.

Grimm, K./Vock, R. (2007): Sozialpädagogik in der Beruflichen Integrationsförderung. Münster u.a.: Waxmann.

Hartmann, H. (1968): Arbeit, Beruf, Profession. In: Soziale Welt, 19. Bd., H. 2, S. 197-212.

Helsper, W. (1996): Antinomien des Lehrerhandelns in modernisierten pädagogischen Kulturen. In: Combe, A./Helsper, W. (Hrsg.): Pädagogische Professionalität. Frankfurt a.M.: Suhrkamp, S. 521-569. 
Hesse, H. A. (1972): Berufe im Wandel: ein Beitrag zur Soziologie des Berufs, der Berufspolitik und des Berufsrechts. Stuttgart: Enke, 2. Aufl.

Huisinga, R./Lisop, I. (1999): Wirtschaftspädagogik. Ein interdisziplinär orientiertes Lehrbuch. München: Vahlen.

Jahn, R.W. (2012a): Stützlehrer in der beruflichen Integrationsförderung. Erarbeitung eines Aufgabenprofils auf Basis berufsanalytischer Verfahren. In: Ulmer, P./Weiß, R./Zöller, A. (Hrsg).: Berufliches Bildungspersonal - Forschungsfragen und Qualifizierungskonzepte. Bielefeld: Bertelsmann, S. 183-202.

Jahn, R.W. (2012b): Stützlehrer Stützlehrer als pädagogische Profession in der Beruflichen Integrationsförderung?! Eine berufs- und professionstheoretische Analyse der Tätigkeit und Entwicklung eines Strukturmodells professioneller pädagogischer Kompetenz. Jena: Friedrich-Schiller-Universität Jena.

Jahn, R.W./Herzog, F. (2011): Berufliche Aufgaben von Stützlehrern in der Beruflichen Integrationsförderung. Eine ethnographische orientierte berufswissenschaftliche Aufgabenanalyse. Jena: [Jenaer Arbeiten zur Wirtschaftspädagogik, 26].

Jahn, R.W./Schmidt, J./Blume, C. (2011): Kooperationsaufgaben von Stützlehrern im Spannungsfeld von Professionalisierung und strukturellen Rahmenbedingungen. In: BWP@ Spezial 5. Online: http:/www.bwpat.de/ht2011/ws10/jahn_etal_ws 10-ht2011.pdf (26.02.2013).

Nieke, W. (2002): Kompetenz. In: Otto, H./Rauschenbach, T./Vogel, P. (Hrsg.): Erziehungswissenschaft. Opladen: Leske + Budrich, S. 13-28.

Oevermann, U. (1996): Theoretische Skizze einer revidierten Theorie professionalisierten Handelns. In: Combe, A./Helsper, W. (Hrsg.): Pädagogische Professionalität. Frankfurt a.M.: Suhrkamp, S. 70-182.

Oser, F. (1997): Standards in der Lehrerbildung. In: Beiträge zur Lehrerbildung, 15. Bd., H. 1, S. 26-37.

Schanne, R. (1990): Berufsanalyse als Weg zur fundierten Berufsorientierung. Nürnberg: IAB.

Schütze, F. (1996a): Überlegungen zu Paradoxien des professionellen Lehrerhandelns in den Dimensionen der Schulorganisation. In: Helsper, W. (Hrsg.): Schule und Gesellschaft im Umbruch. Weinheim: Dt. Studienverlag, S. 333-377.

Sektion für Berufs- und Wirtschaftspädagogik (2009): Memorandum zur Professionalisierung des Personals in der Beruflichen Integrationsförderung aus berufswissenschaftlicher Sicht. Bonn: Pahl-Rugenstein. 